\title{
Self-Triggered and Event-Triggered Set-Valued Observers
}

\author{
Daniel Silvestre, Paulo Rosa, João P. Hespanha, Carlos Silvestre
}

\begin{abstract}
This paper addresses the problem of reducing the required network load and computational power for the implementation of Set-Valued Observers (SVOs) in Networked Control System (NCS). Event- and self-triggered strategies for NCS, modeled as discrete-time Linear Parameter-Varying (LPV) systems, are studied by showing how the triggering condition can be selected. The methodology provided can be applied to determine when it is required to perform a full ("classical") computation of the SVOs, while providing low-complexity state overbounds for the remaining time, at the expenses of temporarily reducing the estimation accuracy. As part of the procedure, an algorithm is provided to compute a suitable centrally symmetric polytope that allows to find hyper-parallelepiped and ellipsoidal overbounds to the exact set-valued state estimates calculated by the SVOs. By construction, the proposed triggering techniques do not influence the convergence of the SVOs, as at some subsequent time instants, set-valued estimates are computed using the conventional SVOs. Results are provided for the triggering frequency of the self-triggered strategy and two interesting cases: distributed systems when the dynamics of all nodes are equal up to a reordering of the matrix; and when the probability distribution of the parameters influencing the dynamics is known. The performance of the proposed algorithm is demonstrated in simulation by using a time-sensitive example.
\end{abstract}

\section{Index Terms}

State estimation; Fault detection; Self-triggered; Networked Control Systems.

\section{INTRODUCTION}

In the context of distributed systems and Networked Control Systems (NCSs), the performance bottleneck is often located in the communication network, either due to low bandwidth, competition for access to a shared medium of communication, or because the network is much slower than the remaining components of the control loop. In distributed systems, different nodes are typically running an algorithm to achieve a certain goal and are often designed to use information from their communicating neighbors. In networked control systems, sensors might be spatially spread over a region of interest and, therefore, measurements have to be sent to a controller/observer over the network. In any of such cases, the network resources are valuable and the communication issues must be considered as they can prevent the stability as given in [32] and [37]. For further details on this topic, the reader is referred to the detailed survey in [31], [16], [36] and [13]; and the book [6].

In the control community, two main strategies have emerged to reduce the communication overhead, namely: event triggering, where the sensor decides, based on the current measurements, if it should transmit to the controller/observer the measured quantities; self triggering, where the controller/observer decides, based on the current estimate of the state, when the sensor should perform the next measurement. An event-triggered solution results in a more informed choice, since the sensor has access to the actual measurement, but prevents the sensor from being shut down between updates. For a recent discussion on event- and self-triggered control and estimation, the reader is referred to [15].

The problem of state estimation for general discrete-time Linear Parameter-Varying (LPV) systems relates to that of determining the set of possible future state values for a given set of inputs, initial state, measurements, and (deterministic) bounds on the noise and disturbances affecting the system. LPV models allow for considering NCSs with parametric uncertainty that may arise from incomplete knowledge of the physical parameters of the processes to be controlled. In the context of distributed observer-based control strategies, uncertainty may also arise due to node heterogeneity or the inability to determine at the observer side which nodes are communicating or taking actions upon the plant. Two interesting instances of the state estimation problem can be found in the following contexts:

- asynchronous distributed algorithms - determining the state of each of the nodes given partial measurements and knowledge of the whole system dynamics;

- networked control systems - the observer must generate an estimate of the state and decide when to require a sensor update or define event conditions for the sensors to take that decision.

Throughout this paper, we focus on two main applications to motivate the theoretical developments of the Set-Valued Observers (SVOs): the use of SVOs to obtain set-valued state estimates of event- and self-triggered networked control systems; and their use for fault detection in randomized distributed systems. They are intrinsically related in the sense that, in both cases, the goal is to minimize either the sensor updates or the computational burden associated with the set-valued computations, in order to reduce the overall cost of implementation of this method in such systems.

Contact the author D. Silvestre through dsilvestredisr.ist.utl.pt for a free unformatted copy of this article or go to https://www.sciencedirect.com/science/article/pii/S0020025517310253 for the final formatted version. 
Fault Detection and Isolation (FDI) has been a long-standing research topic, since the early 70's (see [34]), but still poses remarkable challenges to both the scientific community and the industry (see, for example, the survey in [17] and references therein). Classical fault detection methods such as the ones proposed in [34], [8], [12] and [23], rely on the design of filters that should be able to generate large enough residuals under faulty environments. These strategies aim to derive bounds (or thresholds) on these residuals that can be used to decide whether a fault has occurred or not. However, the calculation of these thresholds is typically cumbersome or poses stringent assumptions on the exogenous disturbances and measurement noise acting upon the plant. In contrast, SVOs aim to compute a set-valued estimate of the state under mild assumptions such as the existence of an overbound for all the signals in the system.

In the context of fault detection, focus is given to the special case of randomized distributed algorithms (see [29] for details on fault detection in this class of systems), for two reasons: their relevance in certain problems - applications range from selection and sorting [22] to consensus [9] and solving high-complexity problems; and, because of their unstructured nature, i.e., all nodes play the same role in the algorithm, while the messages need not satisfy any particular type of time sequence, since any two messages are regarded as having the same purpose. Detecting faults in a distributed way in this setup may lead to a persistent computational and communication overhead, while a self- or event-triggered strategy may yield similar results with far fewer computational and network requirements by running the procedure to obtain the set-valued estimates only when the updates can contribute to the detection.

Within the aforementioned framework, this paper is concerned with obtaining set-valued estimates of the state of the system that are guaranteed to contain the actual state. The approach of using SVOs first introduced in [35] and [26], is adopted - further details can be found in [27], [20] and the references therein. The SVO paradigm has the advantage of posing mild assumptions on the system, while allowing for the computation of a priori bounds for the maximum error. However, the computational cost is still one of the main issues associated with using SVOs (see [10]) although some progress has already been achieved in [30] and [28]. In the remainder of this paper, this limitation will also be tackled by resorting to the use of event- and self-triggered strategies.

The adoption of a mathematical formulation for representing the set of possible states entails the need for fast and nonconservative intersections and unions of sets, as those are the major time-consuming operations when implemented in a computer. An alternative would be to use the concept of zonotopes, described in [7] and further developed in [11] and [1]. However, these represent a different compromise between the speed of the unions and intersections, with the intersections requiring more computations and introducing conservatism. Alternatively, the idea of interval analysis [21] may also be adopted, although it introduces conservatism by not considering higher horizon values in their formulation, unlike the SVOs [24]. Any set-based approach differs from other methods, such as those employing, for example, $H_{\infty}$ filters [33], in that it provides all possible values of the system state that are compatible with the measurements, which is ideal for the implementation of eventtriggered strategies obviating the need for defining threshold values, because the set itself produced by the SVOs represents the event condition for triggering an update.

The strategy for an observer to self-trigger a sensor measurement based on its estimates can resort to an optimization over the update patterns such as in [5], where the disturbances and noise are assumed to be Gaussian. In [3] and [4], Kalman-like filters are proposed for state estimation, thus not providing a deterministic bound for the error. For event-triggered systems, a triggering condition can be posed on the norm of the estimation error being below a given threshold, dependent on the norm of the state [19] [2]; requiring the derivative of a Lyapunov function of the state being semi-negative definite [15], [18]; or, having the norm of the state below a certain threshold [14].

The aforementioned methods can be organized into three groups: algorithms that decide on when to transmit data based on some information about the probability distribution of the state (i.e., using, for example, the covariance matrix produced by the Kalman filter); methods that run an optimization over the possible trigger patterns; and algorithms that perform that decision based on some energy measure of the state (Lyapunov-like and norms under thresholds). These three categories of solutions differ from the current proposal. For the first type, the main difference lies on the use of worst-case set-valued estimates instead of probabilistic filters. The second one involves a complex optimization, as opposed to the greedy approach proposed for the SVOs. The last group of solutions based on norms or Lyapunov functions often differ from a polytopic definition for triggers in the sense that compromise accuracy to gain in performance.

From the perspective of computational load, a Kalman filter solution is attractive due to its light complexity, but does not provide worst-case guarantees as the decision relies on the probability distribution of the state. In addition, designing event-triggered strategies is a non-trivial task, since triggering is based on a threshold imposed to the variance and not on the particular measurements. The remaining strategies revolve around the concept of measuring the energy of the state in some way. These are connected to an SVO-based approach in the sense that both define sets of admissible state values and otherwise a trigger is generated. There is an inherent trade-off between accuracy and complexity. In particular, for LPV systems, a better accuracy provided by the SVOs represents a higher computational cost, but it might also enable a triggering strategy that demands fewer sensor updates.

In this paper, event- and self-trigger strategies are investigated for networked control systems with the objective of developing an online strategy based on set-valued estimates, which means that, at each time instant, the observer produces a polytope, to which the state is guaranteed to belong, and either triggers or allows the sensor to decide the next time instant to perform a 
measurement update.

The class of problems herein addressed poses challenges to the state estimation scheme since, due to the random behavior of gossip algorithms or the network medium, for each possible sensor transmission, the state can belong to a set of possible state realizations originated by the dynamics and the previous state. To consider the worst-case scenario, one needs to perform the union of all possible state sets, which, in general, returns a non-convex set [25]. Furthermore, the number of sets grows exponentially with the number of past time instants considered, i.e., the horizon $N$. As a result, appropriate tools must be employed to reduce this complexity.

The main contributions of this paper can be summarized as follows:

- Given a specific structure for the matrix defining the polytope (i.e., the set-valued state estimate), it is shown how to compute an overbounding hyper-parallelipiped, ellipsoid, or ball;

- Based on the concept of singular vectors, we show how a rotation can be found to prevent the approximation error of using boxes from going to infinity when the matrix defining the polytope is ill-conditioned;

- For the special case of a distributed linear algorithm with a gossip property, it is shown that the overbounds are efficient to compute and propagate, since its complexity is constant;

- It is described how the set-valued state estimates provided by the SVOs can be used to define event- and self-triggering conditions for NCS;

- An algorithm is introduced that uses overbounding methods to approximate the optimal SVO estimates, which is less computationally demanding, and event- and self-triggers the computation of the aforementioned estimates only when necessary to ensure convergence;

- Results are provided regarding the worst-case frequency of the triggers for a class of LPV systems and its probabilistic counterpart when the distribution of the model uncertainties is known a priori;

- Finally, it is given an improved result for convergence that takes into consideration the structure of the output equation of the LPV system.

\section{REFERENCES}

[1] T. Alamo, J. Bravo, and E. Camacho, "Guaranteed state estimation by zonotopes," Automatica, vol. 41, no. 6, pp. 1035 - 1043, 2005.

[2] J. Almeida, C. Silvestre, and A. Pascoal, "Synchronization of multiagent systems using event-triggered and self-triggered broadcasts," IEEE Transactions on Automatic Control, vol. 62, no. 9, pp. 4741-4746, Sept 2017.

[3] - "Self-triggered output feedback control of linear plants in the presence of unknown disturbances," Automatic Control, IEEE Transactions on, vol. 59, no. 11, pp. 3040-3045, Nov 2014.

[4] - "Self-triggered state-feedback control of linear plants under bounded disturbances," International Journal of Robust and Nonlinear Control, vol. 25, no. 8, pp. 1230-1246, 2015. [Online]. Available: http://dx.doi.org/10.1002/rnc.3138

[5] D. Antunes and W. Heemels, "Rollout event-triggered control: Beyond periodic control performance," Automatic Control, IEEE Transactions on, vol. 59, no. 12, pp. 3296-3311, Dec 2014.

[6] A. Bemporad, M. Heemels, and M. Johansson, Networked control systems. Springer, 2010 , vol. 406.

[7] D. Bertsekas and I. Rhodes, "Recursive state estimation for a set-membership description of uncertainty," IEEE Transactions on Automatic Control, vol. 16, no. 2, pp. $117-128$, apr 1971.

[8] J. Bokor and G. Balas, "Detection filter design for LPV systems - a geometric approach," Automatica, vol. 40, pp. 511-518, 2004.

[9] S. Boyd, A. Ghosh, B. Prabhakar, and D. Shah, "Randomized gossip algorithms," IEEE Transactions on Information Theory, vol. 52, no. 6, pp. 2508 2530, Jun. 2006.

[10] P. Casau, P. Rosa, and C. Silvestre, "FITBOX - a Fault Isolation Toolbox," IFAC-PapersOnLine, vol. 48, no. 21, pp. 283 - 288, 2015, 9th IFAC Symposium on Fault Detection, Supervision and Safety for Technical Processes SAFEPROCESS.

[11] C. Combastel, "A state bounding observer for uncertain non-linear continuous-time systems based on zonotopes," in 44th IEEE Conference on Decision and Control, 2005 and 2005 European Control Conference. CDC-ECC '05., dec. 2005, pp. 7228 - 7234.

[12] G. Ducard, Fault-tolerant Flight Control and Guidance Systems: Practical Methods for Small Unmanned Aerial Vehicles, ser. Advances in industrial control. Springer, 2009.

[13] X. Ge, F. Yang, and Q.-L. Han, "Distributed networked control systems: A brief overview," Information Sciences, vol. 380 , pp. 117 - 131 , 2017. [Online]. Available: http://www.sciencedirect.com/science/article/pii/S0020025515005551

[14] W. P. M. H. Heemels, J. H. Sandee, and P. P. J. V. D. Bosch, "Analysis of event-driven controllers for linear systems," International Journal of Control, vol. 81, no. 4, pp. 571-590, 2008. [Online]. Available: http://dx.doi.org/10.1080/00207170701506919

[15] W. Heemels, K. H. Johansson, and P. Tabuada, "An introduction to event-triggered and self-triggered control." in 51st IEEE Conference on Decision and Control, 2012, Maui, HI, USA, pp. 3270-3285.

[16] J. P. Hespanha, P. Naghshtabrizi, and Y. Xu, “A Survey of Recent Results in Networked Control Systems," Proceedings of the IEEE, vol. 95, no. 1, pp. 138-162, Jan. 2007. [Online]. Available: http://dx.doi.org/10.1109/jproc.2006.887288

[17] I. Hwang, S. Kim, Y. Kim, and C. Seah, "A survey of fault detection, isolation, and reconfiguration methods," Control Systems Technology, IEEE Transactions on, vol. 18, no. 3, pp. $636-653$, may 2010.

[18] M. Mazo and P. Tabuada, "On event-triggered and self-triggered control over sensor/actuator networks," in Decision and Control, 2008. CDC 2008. 47th IEEE Conference on, Dec 2008, pp. 435-440.

[19] - "Decentralized event-triggered control over wireless sensor/actuator networks," Automatic Control, IEEE Transactions on, vol. 56, no. 10, pp. 2456-2461, Oct 2011.

[20] M. Milanese and A. Vicino, "Optimal estimation theory for dynamic systems with set membership uncertainty: An overview," Automatica, vol. 27, no. 6, pp. $997-1009,1991$.

[21] R. E. Moore, Interval analysis, ser. Prentice-Hall series in automatic computation. Englewood Cliffs, NJ: Prentice-Hall, 1966.

[22] R. Motwani and P. Raghavan, Algorithms and Theory of Computation Handbook. Chapman \& Hall/CRC, 2010, ch. Randomized Algorithms, pp. 12-12. [Online]. Available: http://dl.acm.org/citation.cfm?id=1882757.1882769

[23] S. Narasimhan, P. Vachhani, and R. Rengaswamy, "New nonlinear residual feedback observer for fault diagnosis in nonlinear systems," Automatica, vol. 44, pp. 2222-2229, 2008. 
[24] P. Rosa and C. Silvestre, "Fault detection and isolation of LPV systems using set-valued observers: An application to a fixed-wing aircraft," Control Engineering Practice, vol. 21, no. 3, pp. 242 - 252, 2013.

[25] P. Rosa, C. Silvestre, and M. Athans, "Model falsification using set-valued observers for a class of discrete-time dynamic systems: a coprime factorization approach," International Journal of Robust and Nonlinear Control, vol. 24, no. 17, pp. 2928-2942, 2014. [Online]. Available: http://dx.doi.org/10.1002/rnc.3033

[26] F. Schweppe, "Recursive state estimation: Unknown but bounded errors and system inputs," IEEE Transactions on Automatic Control, vol. 13, no. 1, pp. $22-28$, feb 1968.

[27] F. Schweppe., Uncertain Dynamic Systems. Prentice-Hall, 1973.

[28] D. Silvestre, P. Rosa, J. P. Hespanha, and C. Silvestre, "Fault detection for lpv systems using set-valued observers: A coprime factorization approach," Systems \& Control Letters, vol. 106, no. Supplement C, pp. 32 - 39, 2017. [Online]. Available: http: //www.sciencedirect.com/science/article/pii/S0167691117301019

[29] _ _ "Stochastic and deterministic fault detection for randomized gossip algorithms," Automatica, vol. 78, pp. 46 - 60, 2017. [Online]. Available: http://www.sciencedirect.com/science/article/pii/S0005109816305192

[30] — "Set-based fault detection and isolation for detectable linear parameter-varying systems," International Journal of Robust and Nonlinear Control, pp. n/a-n/a, 2017, rnc.3814. [Online]. Available: http://dx.doi.org/10.1002/rnc.3814

[31] Y. Tipsuwan and M.-Y. Chow, "Control methodologies in networked control systems," Control Engineering Practice, vol. 11, no. 10, pp. 1099 - 1111, 2003, special Section on Control Methods for Telecommunication. [Online]. Available: http://www.sciencedirect.com/science/article/pii/ S0967066103000364

[32] G. C. Walsh, H. Ye, and L. G. Bushnell, "Stability analysis of networked control systems," IEEE Transactions on Control Systems Technology, vol. 10, no. 3, pp. 438-446, May 2002.

[33] Y. Wei, J. Qiu, H. R. Karimi, and M. Wang, "Filtering design for two-dimensional markovian jump systems with state-delays and deficient mode information," Information Sciences, vol. 269, pp. 316 - 331, 2014. [Online]. Available: http://www.sciencedirect.com/science/article/pii/ S0020025513008980

[34] A. S. Willsky, "A survey of design methods for failure detection in dynamic systems," Automatica, vol. 12, no. 6, pp. 601 - $611,1976$.

[35] H. Witsenhausen, "Sets of possible states of linear systems given perturbed observations," IEEE Transactions on Automatic Control, vol. 13, no. 5, pp. $556-558$, oct 1968 .

[36] X. M. Zhang, Q. L. Han, and X. Yu, "Survey on recent advances in networked control systems," IEEE Transactions on Industrial Informatics, vol. 12, no. 5, pp. 1740-1752, Oct 2016.

[37] X. Zhao, C. Ma, X. Xing, and X. Zheng, "A stochastic sampling consensus protocol of networked euler-lagrange systems with application to two-link manipulator," IEEE Transactions on Industrial Informatics, vol. 11, no. 4, pp. 907-914, Aug 2015. 